\title{
Could intervention by clinics such as Primeros Pasos affect the prevalence of childhood malnutrition in the developing world?
}

\author{
Adam Seccombe ${ }^{1, *}+$ and Elizabeth Hughes ${ }^{2}$ \\ ${ }^{1}$ Post-Graduate Centre, City Hospital, Dudley Road, Birmingham B18 7QH, UK: ${ }^{2}$ Biochemistry Department, \\ Sandwell and West Birmingham Hospitals, Lyndon, West Bromwich, West Midlands, B71 2HJ, UK
}

Submitted 3 September 2007: Accepted 7 January 2009: First published online 27 March 2009

\begin{abstract}
Objective: Guatemala is a country severely affected by malnutrition, particularly in its child population. The present study aimed to assess the impact of recognised aetiological factors on the prevalence of malnutrition and to establish the efficacy of a Guatemalan malnutrition clinic by examining the changes in the prevalence of malnutrition in the ten surrounding communities served by the clinic.

Design: Records of children ( $n$ 7681) aged $5-16$ years seen by the clinic between 2004 and 2006 were examined. Anthropometric data for each child were taken from these records and used as an indicator for their nutritional status to estimate the change in rates of malnutrition during this period.

Results: An overall reduction was seen in all forms of malnutrition over the 3-year period, although over $50 \%$ of the study population remained malnourished in 2006. A consistent reduction was also seen in rates of moderate and severe malnutrition, but not in rates of mild malnutrition.

Conclusions: The results demonstrate that clinics such as Primeros Pasos can have a beneficial effect on schoolchildren, particularly those who are severely malnourished. A need has been highlighted for more research in this area, particularly how clinics such as Primeros Pasos can be best used in the fight against malnutrition.
\end{abstract}

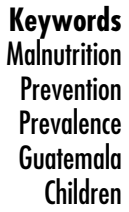

Keywords Prevention Prevalence Children
Guatemala is a country severely affected by malnutrition. Roughly $49 \%$ of all children in Guatemala under the age of 5 years are chronically malnourished ${ }^{(1,2)}$. This is the highest prevalence in Latin America and the fourth highest prevalence in the world ${ }^{(3)}$. A major strategy employed to combat this malnutrition epidemic has been the establishment of volunteer-run clinics aimed at schoolchildren and funded by charitable organisations. One such clinic is Primeros Pasos ${ }^{(4)}$. It was set up in 2004 with the aim of improving malnutrition in the rural communities surrounding Quetzeltenango, a city in the western highlands of Guatemala. The clinic used two methods to improve the nutritional health of the attending children. First, each child underwent a medical examination, including the examination of a stool sample for parasites. If the children were identified as being ill, malnourished or infected, the medical practitioner prescribed treatments, including simple prevention measures. Second, every child attended a health education class run by the clinic to teach them basic hygiene and nutrition.

A study by Pelletier et $a l^{(5)}$ aimed to quantify the impact of malnutrition in disease. They found that malnutrition

† Correspondence address: 56 Farnham Road, Guildford, Surrey, GU2 $4 \mathrm{PE}, \mathrm{UK}$ was an associated cause in approximately $55 \%$ of all deaths in children younger than 5 years of age and that the majority (76-89\%) of these deaths involved mild to moderate malnutrition. Their findings highlight the impact of malnutrition on childhood disease and the importance of treating all forms of malnutrition, including the milder forms. Malnutrition augments the morbidity of disease by lowering the body's ability to resist infection, ultimately leading to longer, more severe and more frequent illnesses in the malnourished child ${ }^{(6)}$. The diseases affected by malnutrition in this way include enteric infection causing diarrhoea, which is endemic among communities in Guatemala $^{(7)}$. For this reason, nutritional interventions have been recognised to be an important approach in reducing mortality from diarrhoea in particular ${ }^{(8)}$. These interventions are especially effective in the developing world as they are cheap and easy to administer. Interventional studies $^{(9)}$ in Guatemala have shown that a simplified medical system relying on local personnel with limited training, combined with nutritional supplements for mothers and infants at high risk, can substantially reduce infant mortality at reasonable cost.

While there is much evidence describing the impact and effective treatment of malnutrition using these volunteer-run clinics in pre-school children ${ }^{(9,10)}$, little 
evidence exists to suggest whether these strategies are also effective in schoolchildren in Guatemala. This is despite the fact that many of the existing programmes in Guatemala only target children in school. In favour of this strategy is substantial evidence in other parts of the world showing that intervention during school years may be able to mitigate the deficits caused by malnutrition, especially cognitive deficiencies ${ }^{(11)}$ and morbidity from disease $^{(8)}$. Because no such research has been carried out in Guatemala, Primeros Pasos and many other clinics throughout the country have had to base their clinical practice upon inappropriate evidence.

The aetiology of childhood malnutrition is highly complicated. It is the result of a sequence of associated events that can be attributed to a wide range of biological, social, cultural and economic factors ${ }^{(12)}$. Some of the socioeconomic factors that have been linked to malnutrition in children seem to act prenatally, such as maternal education level and family income. Other factors, mainly the characteristics of the dwelling and the community, seem to act more directly on the child's nutritional status ${ }^{(13)}$. For Primeros Pasos, which focuses on the health of schoolchildren, it is this latter category that warrants attention. This category includes access to a clean supply of water and the ability to safely dispose of sewage in a sanitary manner. The improvement of both of these factors in particular is universally seen as a critical step in fighting chronic malnutrition ${ }^{(10)}$.

The objectives of the present study were to use previously documented data gathered from the three consecutive years to examine:

1. The prevalence of childhood malnutrition in the ten communities surrounding Primeros Pasos.

2. How this prevalence has changed since Primeros Pasos started its programme of intervention with the children.

3. Whether an association exists between two environmental risk factors (water supply and sewage disposal) and the prevalence of malnutrition in the surrounding communities.

4. How, based on these data, Primeros Pasos could become more effective in the fight against malnutrition in Guatemala.

\section{Materials and methods}

Since 2004, the Primeros Pasos clinic has invited all children from schools in the ten neighbouring communities to attend annually for a medical check-up. The school registers were accepted to be a representative list of the target population for the study: all children aged between 5 and 16 years living in the ten communities surrounding Primeros Pasos. The schools were asked to ensure that every child on their registers attended, whether they were currently attending school or not. Each family was compensated for transport if necessary.
Every child was seen individually by a volunteer at the clinic. These volunteers were all medically trained and were able to speak a high level of Spanish. All children who attended the clinic were Guatemalan and were also able to speak Spanish. Permission was obtained to record and retain any data that were gathered during the consultation. Following the collection of standard demographic data, the child's weight and height were measured, documented and retained by the clinic for the years 2004, 2005 and 2006 for children from each community. Because data were taken from every child who attended the clinic and because all records were included in the study, no sampling method was used.

Data were excluded from the study if any section was incomplete. For the purposes of the present analysis, calculating the child's weight-for-age was seen as the most appropriate method of estimating malnutrition. The degree of malnutrition was determined from the number of standard deviations of the individual's weight from the mean of the relevant reference population, as per the definition of the International Statistical Classification of Diseases and Related Health Problems, 10th edition ${ }^{(14)}$. Any children with a biologically implausible weight-forage were removed from the study, based on exclusion criteria described by the $\mathrm{WHO}^{(15)}$.

The data regarding the children in each community were then illustrated with bar charts to explore the prevalence of malnutrition. Further to this descriptive analysis, the prevalence of malnutrition was compared with statistics describing environmental factors in each community, obtained from a regional census report ${ }^{(16)}$. The association between these variables was examined using correlation analysis as appropriate.

\section{Results}

Data from a total of 7861 children aged 5-16 years were used in the study (2686 in 2004, 2649 in 2005 and 2526 in 2006). Anthropometric and/or demographic data were missing in 131 patient records (104 from 2004, thirteen from 2005 and fourteen from 2006). Three of the 7861 children (all from 2004 data) had biologically implausible values for weight-for-age, based on exclusion criteria described by $\mathrm{WHO}^{(15)}$, and so were removed from the study. Each community had a similar attendance for each of the annual three visits.

As Fig. 1 demonstrates, there was an overall decrease in all three grades of malnutrition and an overall increase in the proportion of children of normal weight between 2004 and 2006. A consistent fall was seen for moderate (2004, 2005 and 2006: $12 \cdot 5 \%, 12 \cdot 3 \%$ and $11 \cdot 1 \%$, respectively) and severe (2004, 2005 and 2006: 0.9\%, $0 \cdot 6 \%$ and $0 \cdot 2 \%$, respectively) malnutrition during this period. This change was less consistent for mild malnutrition (2004, 2005 and 2006: 44.2\%, 46.1\% and 41.2\%, 


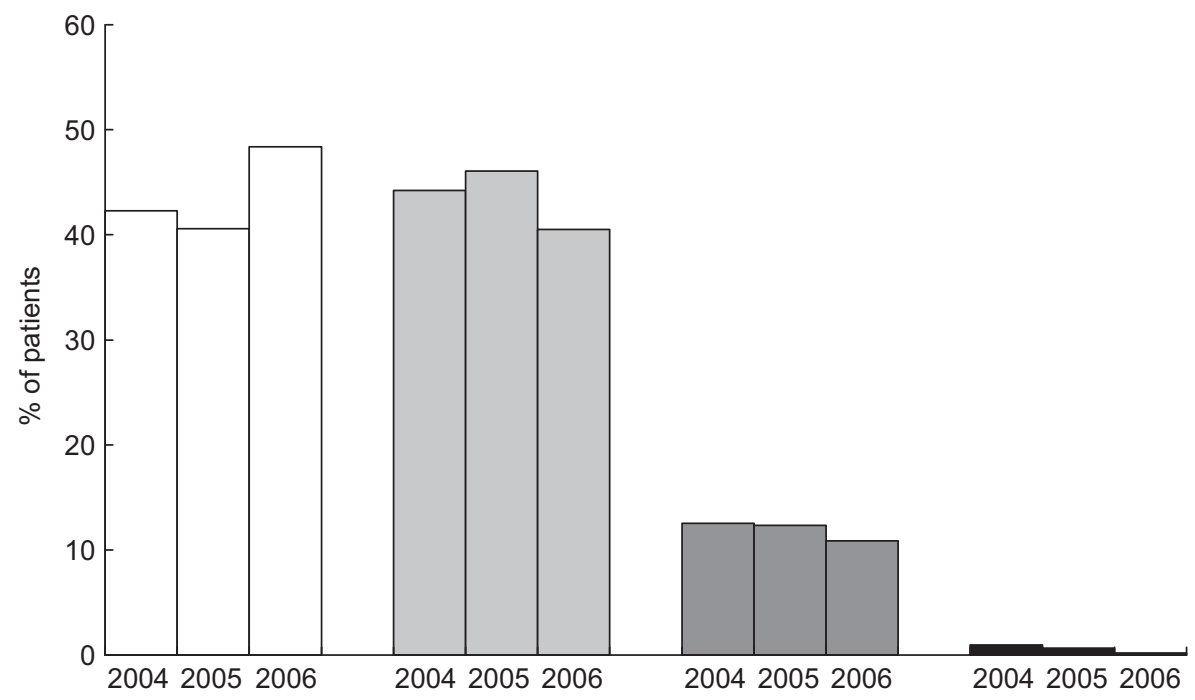

Fig. 1 The change in the prevalence of childhood malnutrition ( $\square$, normal weight; $\square$, mild malnutrition; $\square$, moderate malnutrition; severe malnutrition) in the study population between 2004 and 2006: schoolchildren aged 5-16 years residing in rural areas near Quetzeltenango, Guatemala

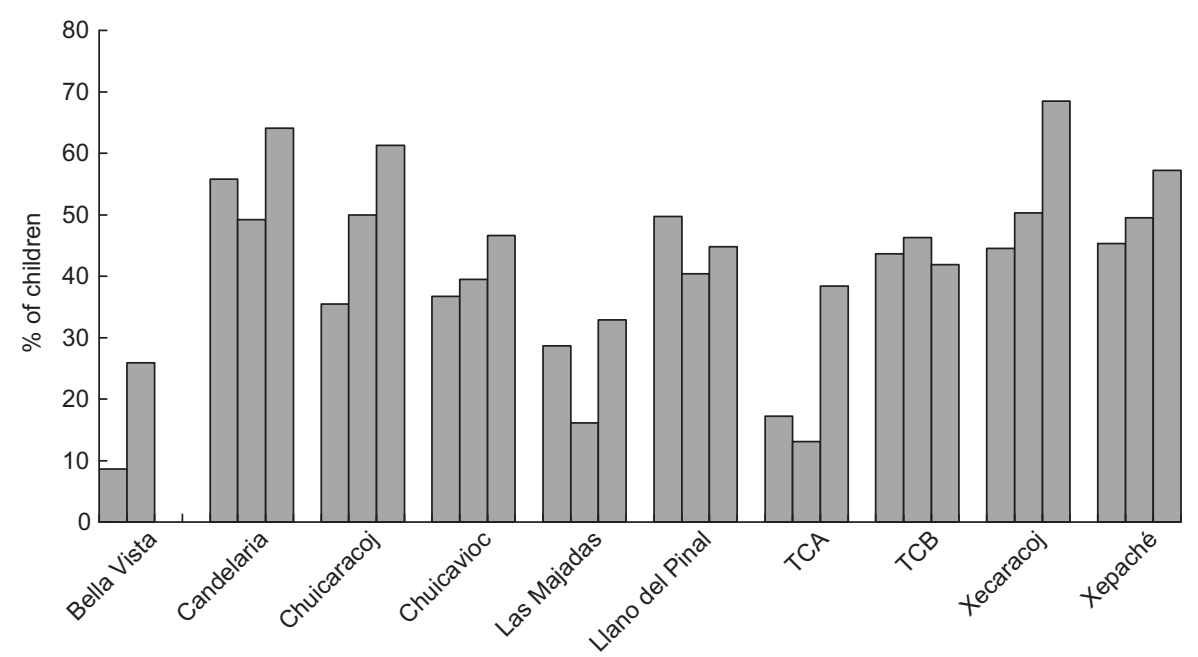

Fig. 2 The proportion of children in each community with normal weight in 2004 (left bar), 2005 (middle bar) and 2006 (right bar): schoolchildren aged 5-16 years residing in rural areas near Quetzeltenango, Guatemala

respectively) and for normal weight (2004, 2005 and 2006: $42 \cdot 3 \%, 40 \cdot 6 \%$ and $47 \cdot 4 \%$, respectively), both showing a small decrease in nutritional health between 2004 and 2005. The improvement in the nutritional status of the children was much more substantial in all groups between 2005 and 2006.

Figures 2 and 3 illustrate the change in nutritional status among each of the ten communities. They demonstrate a significant success of the intervention used by Primeros Pasos in improving the health of children in the ten communities. When the prevalence of malnutrition was examined between the different communities, a large variation was found in the point prevalence at any one time. For example, in 2006 the prevalence of malnutrition in the communities varied between $35 \cdot 9 \%$ and $67 \cdot 1 \%$.
Variation was also seen in the change of prevalence of malnutrition over the 3-year period. Overall most communities showed an improvement in health, the majority (seven) of the communities showing a reduction in malnutrition prevalence. It appeared that the clinic had substantial success in reducing the prevalence of severe malnutrition from those communities in which it was a problem.

Two environmental parameters were examined and compared with the prevalence of malnutrition: the presence of a potable water supply and the method of sewage disposal. The data in Table 1 were taken from the 2000 regional census ${ }^{(16)}$.

Figure 4 illustrates that the communities can be divided into two groups based on their access to a mains water 


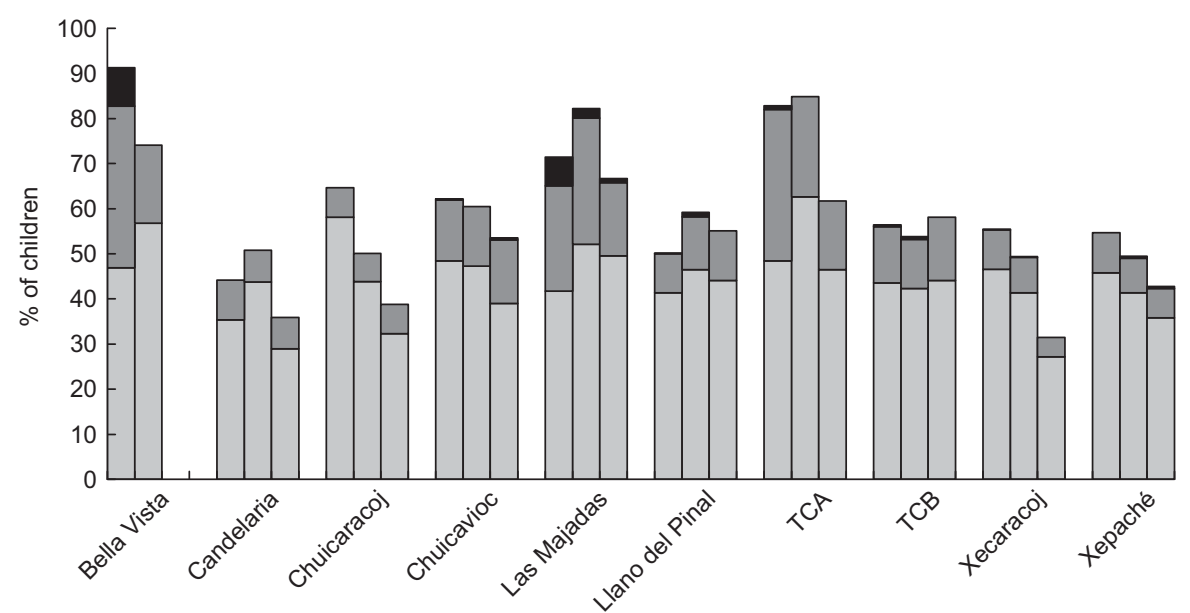

Fig. 3 The proportion of children with varying grades of malnutrition ( $\square$, mild malnutrition; $\square$, moderate malnutrition; $\square$, severe malnutrition) in each community in 2004 (left bar), 2005 (middle bar) and 2006 (right bar): schoolchildren aged 5-16 years residing in rural areas near Quetzeltenango, Guatemala

Table 1 The percentage of homes with water and sewage supply in each community, rural areas near Quetzeltenango, Guatemala, 2004

\begin{tabular}{lcc}
\hline Community & $\begin{array}{c}\text { \% with mains water } \\
\text { supply }\end{array}$ & $\begin{array}{c}\text { \% with a defined method } \\
\text { of sewage removal }\end{array}$ \\
\hline Bella Vista & $90 \cdot 5$ & $71 \cdot 4$ \\
Candelaria & $43 \cdot 2$ & $70 \cdot 5$ \\
Chuicaracoj & $20 \cdot 8$ & $73 \cdot 1$ \\
Chuicavioc & $80 \cdot 3$ & $77 \cdot 9$ \\
Las Majadas & $47 \cdot 5$ & $64 \cdot 7$ \\
Llano del Pinal & $84 \cdot 7$ & $82 \cdot 8$ \\
TCA & 0 & $31 \cdot 7$ \\
TCB & $46 \cdot 6$ & $69 \cdot 8$ \\
Xecaracoj & $68 \cdot 5$ & $78 \cdot 1$ \\
Xepaché & $74 \cdot 4$ & 84.5
\end{tabular}

*Defined as having a sewer, septic tank or enclosed pit for sewage that ensures the sewage is kept separate from both human contact and the water supply.

supply: one with less than $50 \%$ of inhabitants with access and the other with greater than $65 \%$ of inhabitants with access. There was no clear association between access and the prevalence of malnutrition in the former group. The latter group, however, demonstrated a strong negative correlation between the presence of a mains water supply and the nutritional status of the community in terms of the proportion of children of normal weight $(r=-0.979, P<0.05)$.

Figure 5 suggests a small positive impact of adequate sewage removal on the nutritional health of the communities, with a downward trend for malnutrition among children as a higher proportion of homes had a good method of sewage disposal.

\section{Discussion}

The number of children attending from each of the ten communities varied greatly. This is most likely to reflect the size of the communities as opposed to any variation in attendance, assuming the school registers were complete. Since all clinic consultations and treatments were free and because all communities were within a short distance of the clinic, it was felt that no child from any of the ten communities would have been excluded from attending. No parent refused to allow their child's data to be recorded or used by the clinic over the period studied. The data from 2004 were less complete, necessitating the exclusion of more records than in other years. This difference can be attributed to a change of management that took place in 2005 and resulted in a better standardisation of data recording.

The prevalence of malnutrition reflected current views that malnutrition is a severe problem in this part of Guatemala. Over $50 \%$ of the children in the study in 2006 were malnourished. On a more positive note, a reduction in all three levels of malnutrition was seen from the time when the clinic started treating schoolchildren in 2004. While the clinic is likely to be a factor in this gradual improvement in nutritional status, the absence of baseline data from before 2004 makes it difficult to quantify this effect. It may well be that this improvement is more a reflection of decreasing poverty levels in the area or a public health measure introduced by the government. The improvement in the nutritional health of the children was greater for all degrees of malnutrition from 2005 to 2006. This may be a result of a number of factors; the compliance of the children may have improved after multiple visits to the clinic, there may be a lag period between intervention by the clinic and a therapeutic effect in the children, the clinic may have become more effective over the three years or factors external to the clinic may have coincidentally impacted children's health in the area.

The study highlights a threefold reduction in the prevalence of severe malnutrition and a consistent reduction in the prevalence of moderate and severe malnutrition 


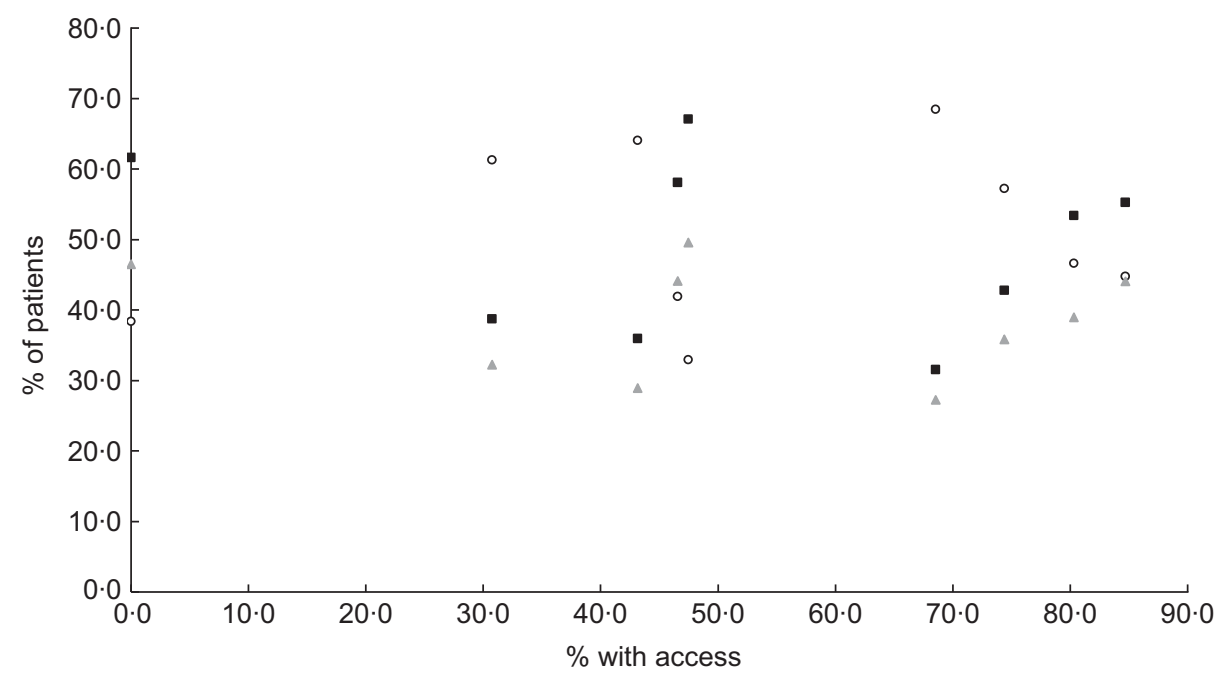

Fig. 4 The relationship between access to a mains water supply and the prevalence of nutritional status $(O$, normal weight; malnourished; $\triangle$, mildly malnourished) in 2006: schoolchildren aged 5-16 years residing in rural areas near Quetzeltenango, Guatemala

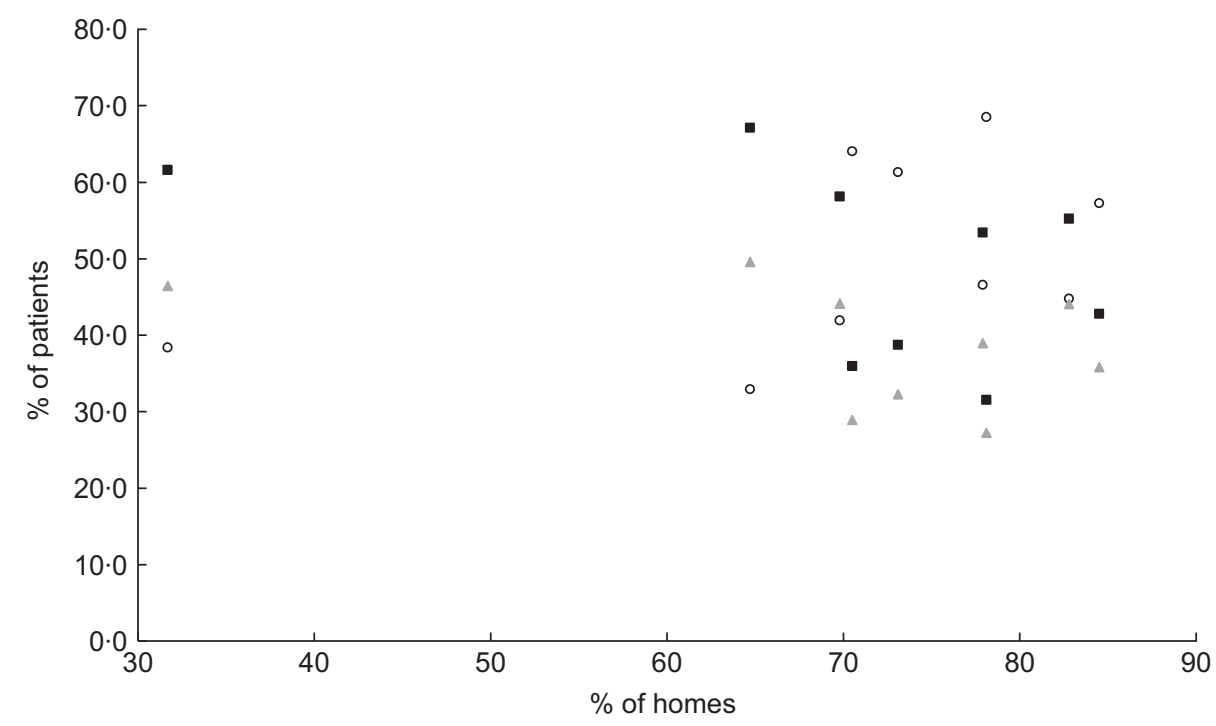

Fig. 5 The relationship between having a method of sewage disposal and the prevalence of nutritional status $(O$, normal weight; , malnourished; $\triangle$, mildly malnourished) in 2006: schoolchildren aged 5-16 years residing in rural areas near Quetzeltenango, Guatemala

over the three years. While there was also a reduction in the rates of mild malnutrition, it was both less sizeable and less consistent. This may suggest that the clinic pays more attention to treating moderate and severe degrees of malnutrition compared with the milder forms. Since the moderately and severely malnourished children would have appeared more severely unwell, the clinic may have consciously or unconsciously treated this cohort more intensively. Because of the large proportion of complications caused by mild and moderate malnutrition, however, strategies that concentrate on the severely malnourished will do little to address the impact of malnutrition on a community $^{(17)}$. Alternatively, this difference in efficacy may indicate that a proportion of the damage from malnutrition has already occurred in early childhood and the clinic can do little to reverse it. The low weight-for-age seen in these children may simply reflect a more permanent stunting that has occurred earlier in life, which nutritional supplements or improvements in diet can no longer fully correct.

The variance in point prevalence of malnutrition that was seen between the ten communities is likely to reflect a variance in the presence or impact of the many aetiological factors for malnutrition. There was also a discrepancy in the change in malnutrition prevalence over time between the communities. Whether this discrepancy is due to alterations in environment and behaviour 
between the ten communities or due to the clinic having a varying effectiveness in different communities is unclear from the data. Those communities with high levels of severe malnutrition saw a dramatic fall in its prevalence over the period. This may reflect the success of the clinic in treating children with a severe pathology compared with a milder pathology for the reasons mentioned above. It may also be due to the differences in data recording between 2004 and 2005/2006.

There are many environmental factors that impact on malnutrition. Of these key factors, two parameters were examined: access to a mains water supply and access to a defined method of sewage removal. When the relationship between having access to a mains water supply and the nutritional status of the community was examined, a significant negative association was found for those communities with greater than $65 \%$ of inhabitants having access to mains water. This seems unusual as one would expect an improvement in nutritional health in communities with greater access to a mains water supply. Within this reasoning however, there is an assumption that the mains water will be safe to drink. While many studies recognise the importance of increasing the availability of water to reduce rates of malnutrition ${ }^{(10)}$, it is equally important to ensure the quality of the water supply. The Guatemalan Ministry of Health has recently reported that $98 \%$ of the country's water sources are contaminated in some way ${ }^{(18)}$. With this in mind, if the move towards a mains water supply is encouraging the population to discontinue their practice of sterilising water before consumption, then it may actually be having a deleterious effect. Data regarding sewage removal suggested a slight positive but insignificant impact of better sewage removal on reducing malnutrition prevalence. This slight effect may hint at a reduced risk of contaminating the water supply or may be linked to other factors associating with having better sewage disposal, such as improved hygiene behaviour, which would also reduce malnutrition prevalence.

The use of pre-existing data from the clinic allowed for a convenient and efficient analysis of large numbers of records. The opportunity for observer bias was diminished as the results were collected by people other than the researcher. Furthermore, all parameters used in the study were objective measurements and so were unaffected by cultural or language barriers that may have been encountered. However, it was accepted that there were some differences in the accuracy of data recording between the three years, with the 2004 data being less complete and having less rigid protocols in place for data acquisition. This may have led to a recording bias. Inconsistencies in spelling of names and lack of another means of identification made recognition of the same child across different years extremely difficult. This prevented further statistical analyses from being carried out which would have added more weight to the conclusions. To resolve this issue and to improve continuity of care, the clinic may wish to consider the use of patient identification numbers.

The present study has provided evidence to show that clinics such as Primeros Pasos can have an effect in schoolchildren. Further research using a control group is needed to clarify both the existence and the magnitude of this effect. These data indicated that the efficacy of the clinic appeared to be particularly beneficial for children who were severely malnourished, suggesting that the clinic can play an important role in preventing the health of already malnourished children from deteriorating and rescuing those whose health has already deteriorated. The evidence does imply, however, that much of the low weight-for-age seen in Guatemala is not easily reversed in older children. More severe forms of malnutrition seemed to reduce in prevalence but milder forms of malnutrition seemed to be harder to reverse. This agrees with conventional belief that the most damaging effects of malnutrition occur before 2 years of age ${ }^{(10)}$. Thus while clinics such as Primeros Pasos may be useful in improving and maintaining the health of children in school, they should be used as adjuncts to programmes that directly target pre-school children. Because the sources of funding for malnutrition clinics are fragmented, with money coming from local and foreign charities and from various government funds, there appears to be no clear strategy to tackling childhood malnutrition in Guatemala. As more data become available from the Primeros Pasos clinic, further research can clarify the capabilities of such clinics and identify their role in the fight against malnutrition. Such research should be used to create a national strategy to tackle malnutrition across Guatemala to ensure that the available funds are most wisely used. Investigation of the energy intake of the children in surrounding communities may further direct intervention.

Regarding the different communities, clear differences in the prevalence of malnutrition have been demonstrated. These differences provide an ideal opportunity to examine and quantify the impact of the differing aetiological factors in each community. The study has highlighted some possible avenues for further research in this area. In particular, urgent research needs to be carried out into the provision of a mains water supply to the communities to determine the quality of the water and the impact on both the health and the behaviour of the target communities. It is hoped that the present study can improve the awareness of these research needs and encourage interest in this critical area. By having created and continuing to create a substantial collection of records regarding malnutrition, Primeros Pasos can play a vital role in providing the data for such future research.

\section{Acknowledgements}

The authors would like to thank the British Medical and Dental Students' Trust, the British Nutrition Foundation 
and the Vandervell Foundation for their financial support. These sponsors had no involvement in the study itself. No author has any conflict of interest. A.S. designed the study, carried out the data analyses and drafted the manuscript. E.H. approved the study design and provided significant advice regarding the drafting of the manuscript. Both authors read and approved the final manuscript.

\section{References}

1. Benson Agriculture and Food Institute (1999) Diagnostic: Assessing the Food and Nutrition Situation in Rural Guatemala. http://benson.byu.edu/Publication/BI/Lessons/ volume21/volume21.htm (accessed October 2006).

2. World Food Programme (2006) Projected 2006 Needs for World Food Programme Projects and Operations: Guatemala. http://www.wfp.org/country_brief/indexcountry.asp? country $=320 \#($ accessed October 2006).

3. UNICEF (2006) The State of the World's Children 2006. http:// www.unicef.org/publications/index_30398.html (accessed October 2006).

4. Primeros Pasos Guatemala (2006) Primeros Pasos homepage. http://www.primerospasos.org (accessed October 2006).

5. Pelletier DL, Frongillo EA Jr, Schroeder DG \& Habicht JP (1994) A methodology for estimating the contribution of malnutrition to child mortality in developing countries. J Nutr 124, Suppl., 2106S-2122S.

6. Scrimshaw NS (2003) Historical concepts of interactions, synergism and antagonism between nutrition and infection. J Nutr 133, Suppl., 316S-321S.

7. Anderson TJ, Zizza CA, Leche GM, Scott ME \& Solomons NW (1993) The distribution of intestinal helminth infections in a rural village in Guatemala. Mem Inst Oswaldo Cruz 88, 53-65.
8. Jamison DT, Breman JG, Measham AR, Alleyne G, Claeson M, Evans DB, Prabhat J, Mills A \& Musgrove P (1993) Disease Control Priorities in Developing Countries. New York: Oxford University Press.

9. Hearst $N$ (1985) Infant mortality in Guatemala: an epidemiological perspective. Int J Epidemiol 14, 575-581.

10. Marini A \& Gragnolati M (2003) Malnutrition and Poverty in Guatemala. World Bank Policy Research Working Paper no. 2967. Washington, DC: World Bank.

11. Tarleton JL, Haque R, Mondal D, Shu J, Farr BM \& Petri WA $\mathrm{Jr}$ (2006) Cognitive effects of diarrhea, malnutrition, and Entamoeba histolytica infection on school age children in Dhaka, Bangladesh. Am J Trop Med Hyg 74, 475-481.

12. Gopalan S (2000) Malnutrition: causes, consequences, and solutions. Nutrition 16, 556-558.

13. Delpeuch F, Traissac P, Martin-Prevel Y, Massamba JP \& Maire B (2000) Economic crisis and malnutrition: socioeconomic determinants of anthropometric status of preschool children and their mothers in an African urban area. Public Health Nutr 3, 39-47.

14. World Health Organization (2006) International Statistical Classification of Diseases and Related Health Problems. http://www3.who.int/icd/currentversion/fr-icd.htm (accessed October 2006).

15. World Health Organization (1995) Physical Status: The Use and Interpretation of Anthropometry. Report of a WHO Expert Committee. WHO Technical Report Series no. 854. Geneva: WHO.

16. Unidad de Investigación, Estadística y Planificación (2000) Recuento Censal Quetzeltenango Mayo-Agosto 2000. Información Estadística y Social del Municipio de Quetzeltenango 2000. Quetzeltenango, Guatemala: Unidad de Investigación, Estadística y Planificación.

17. Pelletier DL, Frongillo EA Jr, Schroeder DG \& Habicht JP (1995) The effects of malnutrition on child mortality in developing countries. Bull World Health Organ 73, 443-448.

18. UNICEF (2005) The Big Picture: Guatemala. Press Release. New York: UNICEF. 